\title{
Magnetoelectric coupling on multiferroic cobalt ferrite-barium titanate ceramic composites with different connectivity schemes
}

ARTICLE in ACTA MATERIALIA · MAY 2015

Impact Factor: 4.47 · DOI: 10.1016/j.actamat.2015.02.032

13 AUTHORS, INCLUDING:

Carolin Schmitz-Antoniak

Forschungszentrum Jülich

39 PUBLICATIONS 367 CITATIONS

SEE PROFILE

\section{Ahmadshah Shahab Nazrabi}

University of Duisburg-Essen

2 PUBLICATIONS 0 CITATIONS

SEE PROFILE
Harsh Trivedi

University of Duisburg-Essen

4 PUBLICATIONS 0 CITATIONS

SEE PROFILE

Vladimir V. Shvartsman

University of Duisburg-Essen

137 PUBLICATIONS 1,975 CITATIONS

SEE PROFILE 


\title{
Magnetoelectric Coupling on Multiferroic Cobalt Ferrite- Barium Titanate Ceramic Composites with Different Connectivity Schemes
}

\author{
Morad Etier ${ }^{1}$, Carolin Schmitz-Antoniak ${ }^{2}$, Soma Salamon ${ }^{3}$, Harsh Trivedi ${ }^{1}$, Yanling Gao ${ }^{1}$, Joachim Landers ${ }^{3}$, \\ Devendraprakash Gautam ${ }^{4}$, Markus Winterer ${ }^{4}$, Detlef Schmitz ${ }^{5}$, Heiko Wende ${ }^{3}$, Vladimir V. Shvartsman ${ }^{1}$, \\ and Doru C. Lupascu ${ }^{1}$ \\ ${ }^{1}$ Institute for Materials Science and Center for Nanointegration Duisburg-Essen (CENIDE), University of Duisburg-Essen, \\ Universitätsstraße 15, 45141 Essen, Germany \\ ${ }^{2}$ Peter Grünberg Institute (PGI-6), Jülich Research Centre, 52425 Jülich, Germany \\ ${ }^{3}$ Faculty of Physics and Center for Nanointegration Duisburg-Essen (CENIDE), University of Duisburg-Essen, Lotharstraße 1, \\ 47057 Duisburg, Germany \\ ${ }^{4}$ Nanoparticle Process Technology, Faculty of Engineering and Center for Nanointegration Duisburg-Essen (CENIDE), University \\ of Duisburg-Essen, Lotharstraße 1, 47057 Duisburg, Germany \\ ${ }^{5}$ Helmholtz-Zentrum für Materialien und Energie, Albert-Einstein-Str. 15, 12489 Berlin, Germany
}

\begin{abstract}
In this article we report on the synthesis and multiferroic properties of cobalt ferrite $\left(\mathrm{CoFe}_{2} \mathrm{O}_{4}\right)$ - barium titanate $\left(\mathrm{BaTiO}_{3}\right)$ biphasic composites. The initial composite nanopowder was synthesized by a combination of co-precipitation and organosol methods. A ceramic sample with (3-0) connectivity, i.e. $\mathrm{BaTiO}_{3}$ grains in a $\mathrm{CoFe}_{2} \mathrm{O}_{4}$ matrix was obtained by a combination of spark plasma sintering and annealing. In order to understand the correlations between morphology, electric properties, and magnetization, we present a detailed study at different preparation steps and compare it to the properties of a conventionally sintered sample with the traditional (0-3) connectivity, i.e. $\mathrm{CoFe}_{2} \mathrm{O}_{4}$ grains in a $\mathrm{BaTiO}_{3}$ matrix. We observe that the (3-0) sample shows improved magnetic properties in comparison to the conventionally sintered composite of same composition. In spite of relatively large leakage current for the (3-0) sample compared to the traditional (0-3) one, it exhibits a converse magnetoelectric effect that follows the $\mathbf{H}_{\mathrm{dc}}$ dependence of the piezomagnetic coefficient. The magnetic fielddependence of electric polarization at the surface was investigated utilizing X-ray absorption spectroscopy and its associated linear and circular dichroisms.
\end{abstract}

Keywords: Mutliferroics; converse magnetoelectric effect; composite; spark plasma sintering 


\section{INTRODUCTION}

Magnetoelectric (ME) materials can be electrically polarized by a magnetic field or magnetized by an electric field. They can be used in different technological applications such as magnetic sensors, microelectromechanical systems (MEMS), and energy harvesters [1-3]. Composite multiferroic materials, which consist of ferroelectric and ferromagnetic phases, show a much larger magnetoelectric effect compared to single-phase materials. In the composites, the magnetoelectric effect is typically generated through a mechanical strain arising under an applied magnetic or electric field at interfaces between the two constituents. Of particular interest are nanoscalestructured materials, where a high density of interfaces may enhance the magnetoelectric coupling. Nano-composites, which exhibit multiferroic, as well as magnetoelectric properties, have been prepared by various methods including solid state reaction, sol-gel, and hydrothermal synthesis $[4,5,6]$. Barium titanate, $\mathrm{BaTiO}_{3}$, is often used as the ferroelectric constituent due to its excellent piezoelectric properties and lead free chemical composition. Cobalt ferrite, $\mathrm{CoFe}_{2} \mathrm{O}_{4}$, is a widespread magnetic component due to its strong magnetostriction. An important advantage of $\mathrm{CoFe}_{2} \mathrm{O}_{4}-\mathrm{BaTiO}_{3}$ composites is the spinodal decomposition of this binary system, which prevents reaction between the constituents during high-temperature processing. It is well documented that ferroelectric properties of $\mathrm{BaTiO}_{3}$ and ferromagnetic properties of $\mathrm{CoFe}_{2} \mathrm{O}_{4}$ depend on particle size [7,8]. Correspondingly, one should expect a size effect on the magnetoelectric coupling in the composites.

To obtain dense ceramics with nanometer grain size the spark plasma sintering (SPS) technique can be used. This technique is based on applying a pressure simultaneously with an electric pulse through the material causing high heating rates. SPS decreases the densification time from hours to minutes, suppresses grain growth, and reduces the diffusion at grain boundaries. The 
SPS method has been used e.g. for barium titanate densification $[9,10]$. Ghosh et al. reported on the synthesis of multiferroic nano-composites $\mathrm{CoFe}_{2} \mathrm{O}_{4}-\mathrm{BaTiO}_{3}$ at weight ratios 10/90 and 20/80 via the SPS technique [11]. They found a reduction of dielectric permittivity with increasing $\mathrm{CoFe}_{2} \mathrm{O}_{4}$ content and related this to $\mathrm{Fe}$ diffusion into the $\mathrm{BaTiO}_{3}$ phase.

The properties of biphasic ferromagnetic/ferroelectric composites, in particular the magnetoelectric (ME) coupling, depend on the type of connectivity between the two constituent phases. In this sense, the connectivity means the number of dimensions through which the material is continuous. For bulk materials, the (0-3) connectivity scheme [12] is typically used, in which ferromagnetic regions are distributed in a ferroelectric dielectric matrix and are well separated from each other, i.e. the ferromagnetic phase has connectivity 0 and the dielectric matrix has connectivity 3. Such a scheme provides sufficient electric resistivity of the composites and facilitates the electric poling process which is necessary to achieve a large ME response. Due to larger conductivity, the multiferroic composites with the (3-0) connectivity (ferroelectric inclusions in a ferromagnetic matrix) are less studied. Nevertheless, they can find applications in devices using switching or modulation of electrical polarization by a magnetic field, materials with magnetically tunable dielectric permittivity, or microwave absorbing materials $[13,14]$. Furthermore, measurements of the ME effect in (3-0) composites provides a different approach for understanding the strain mediated ME effect via interfaces for different connectivity schemes in modeling.

The aim of the present paper is to investigate the multiferroic properties and the ME effect of a $\mathrm{CoFe}_{2} \mathrm{O}_{4}-\mathrm{BaTiO}_{3}$ (3-0) ceramic composite synthesized by the SPS method and compare it to the $(0-3)$ one. 


\section{EXPERIMENTAL TECHNIQUES}

The procedures for the synthesis of the composite nanopowders are described in detail elsewhere [15]. Shortly, the co-precipitation method is utilized to synthesize $40 \mathrm{~nm} \mathrm{CoFe} 2 \mathrm{O}_{4}$ nanoparticles. After that, a colloidal suspension of $\mathrm{CoFe}_{2} \mathrm{O}_{4}$ nanoparticles is prepared using oleic acid and oleylamine $[16,17]$. The obtained ferrofluid is added to the $\mathrm{BaTiO}_{3}$ precursor. The weight percentage of $\mathrm{CoFe}_{2} \mathrm{O}_{4}$ and $\mathrm{BaTiO}_{3}$ components is nominally $50 \%$ each. The two-phase precursor is mixed and then calcined at $750^{\circ} \mathrm{C}$ in a conventional chamber furnace for 15 minutes in order to form a powder (sample S1). Agglomerates are destroyed using ball milling.

The resulting powder was then introduced to a SPS instrument (FCT HP D5, FCT Systeme GmbH, Raunstein, Germany). The powder mixture was loaded into a graphite die with an inner diameter of $20 \mathrm{~mm}$, an outer diameter of $45 \mathrm{~mm}$, and a height of $50 \mathrm{~mm}$. A boron nitride coated graphite foil was used to avoid contact between the powder and the inner surface of the die. This is to ascertain that the current flows through the sample during sintering and not through the die. To minimize radial temperature distribution and radiation heat losses the graphite die was covered by graphite wool. The sample was heated by a pulsed electric current from room temperature to $1000^{\circ} \mathrm{C}$ with a heating rate of $100{ }^{\circ} \mathrm{C} / \mathrm{min}$. The sample was held at $1000^{\circ} \mathrm{C}$ for 5 minutes and then cooled down to $500^{\circ} \mathrm{C}$ with a rate of $100^{\circ} \mathrm{C} / \mathrm{min}$, and further to room temperature by natural cooling. An uniaxial pressure of $35 \mathrm{MPa}$ was applied on the sample over the complete heatingcooling cycle. The pressure on the sample was released during natural cooling. The complete sintering process was performed in vacuum at about 1 mbar. Temperature measurements were carried out with an optical pyrometer focused on the surface of the upper graphite push-punch. The as-sintered ceramics were polished to remove the graphite foil from the surface of the sintered pellet (sample S2). One of the SPS sintered ceramics was annealed at $900^{\circ} \mathrm{C}$ for 2 hours in a normal 
chamber furnace (Nabertherm $\mathrm{GmbH}$ ) (sample S3). For comparison, another sample was prepared by sintering nanoparticle powder normally at $1200^{\circ} \mathrm{C}$ for 2 hours without SPS (sample S4).

The phase content and crystal structure of the composites were analyzed by X-ray diffraction (XRD) (Siemens D5000) while sample morphology was studied by scanning electron microscopy (SEM) (Quanta 400 FEG). Before SEM measurements, the samples were well polished and thermally etched. The program AnalySIS (Soft Imaging Systems) was used for analyzing SEM micrographs and particle/grain sizes determination. The ferroelectric properties were studied locally using piezoresponse force microscopy (PFM) (MFP-3D, Asylum Research) and macroscopically using a self-built Sawyer-Tower circuit. Dielectric characteristics were measured using a Solartron 1260 impedance analyzer with the dielectric interface 1296. For electrical measurements, silver electrodes were painted onto the sample faces. The magnetic measurements were performed by SQUID magnetometry (MPMS-5S, Quantum Design)

Usually the magnetoelectric coupling in composites is characterized by measurements of the direct ME effect (polarization or voltage produced by an applied magnetic field), e.g. by using the dynamic lock-in technique [18]. However, for samples which are poor insulators it is difficult to detect the direct ME effect, because the leakage current will partly short-circuit the sample and reduce the measured ME voltage coefficient. This complication can be circumvented by measuring the converse ME effect (magnetization produced by an electric field). In this case, the electric power supply can compensate the ohmic losses and still provide sufficient electric fields to generate the magnetic signal. Attention must be paid to Joule heating, though. In this study, we performed measurements of the converse ME effect using a custom-built setup based on the AC susceptometer of a SQUID magnetometer [19]. An AC electric field induces an alternating magnetic moment. Its first harmonic is detected using an internal lock-in amplifier. We addressed the longitudinal magnetoelectric effect, where the applied magnetic field, applied electric field, and the measured 
magnetization were parallel to each other and perpendicular to the sample surface. Before the ME measurements the ceramic samples S3 and S4 were poled in silicon oil under an electric field of $12 \mathrm{kV} / \mathrm{cm}$ applied perpendicular to the sample surface at a temperature of $415 \mathrm{~K}$. The electric field was kept as the samples were cooled to room temperature.

X-ray absorption spectroscopy in the soft X-ray regime was performed at the high-field end station at the helical undulator beamline UE46-PGM1, HZB-BESSYII synchrotron radiation facility, by measuring the sample drain current at a temperature of $T=290 \mathrm{~K}$. Magnetic fields up to 1.5 $\mathrm{T}$ were applied either parallel or perpendicular to the $k$ vector of incoming $\mathrm{x}$-rays. For measurements of the $\mathrm{x}$-ray linear dichroism (XLD) at the $\mathrm{Ti} \mathrm{L}_{3,2}$ absorption edges, the photon energy was tuned between $440 \mathrm{eV}$ and $500 \mathrm{eV}$ with a scan speed of $0.35 \mathrm{eV} / \mathrm{s}$ for either horizontally or vertically polarized x-rays. Only small charging effects were observed by monitoring the sample drain current. After about 10min, the signal was quite stable and the measurements were started. Special care was taken to check the possible influence of a tiny energy shift between different x-ray polarizations, which was found to be negligible here. Measurements were performed in grazing incidence $\left(\theta_{k}=60^{\circ}\right)$ with the main component of the magnetic field vector applied perpendicular to the sample surface $\left(\theta_{H}=-30^{\circ}\right)$. The electric field vector for horizontally polarized $\mathrm{x}$-rays was parallel to the magnetic field vector, whereas the electric field vector for vertically polarized x-rays lay in the sample plane. A detailed description and illustration of the measurement geometry can be found elsewhere [20].

Magnetic field-dependent element-specific magnetization curves were measured by detecting the sample drain current for left or right circularly polarized x-rays at the photon energy of maximum x-ray magnetic circular dichroism (XMCD) at the $\mathrm{Fe} \mathrm{L}_{3}$ absorption edge for different values of the external magnetic field. 


\section{RESUlTS AND DisCUSSION}

\subsection{STRUCTURE AND MORPHOLOGY AT DIFFERENT PREPARATION STAGES}

Figure 1 shows XRD results for the composite powder and ceramics sintered by the SPS method. Only $\mathrm{BaTiO}_{3}$ and $\mathrm{CoFe}_{2} \mathrm{O}_{4}$ phases were detected without any trace of other phases or impurities. A pronounced broadening of the Bragg reflections observed for the powder sample indicates that the particle sizes are in the nanometer range. The intensities of $\mathrm{CoFe}_{2} \mathrm{O}_{4} \mathrm{Bragg}$ peaks were much lower than those of $\mathrm{BaTiO}_{3}$. Both for the powder (Fig. 1a) and the ceramic sample sintered by SPS (Fig. 1b), the crystalline structure of $\mathrm{BaTiO}_{3}$ seems to be cubic, since no splitting of the Bragg reflections corresponding to crystallographic planes $(200) /(002)$ and $(112) /(211)$ was observed (see two bottom curves in right panel figure 1d). On the other hand, for the annealed SPS sample (S3) the splitting of the peaks is clearly visible, which indicates that $\mathrm{BaTiO}_{3}$ is in a tetragonal and hence ferroelectric state [21]. The larger intensity of the (112) peak at smaller angles compared to the (211) peak at larger angles indicates that the sample has a texture with the preferential c-axis alignment perpendicular to the sample surface. A similar ratio between (112) and (211) peaks was observed for the conventionally sintered composite ceramics.

Figure 2 compares the morphology for different samples. The as prepared powder S1 showed particles distributed in a narrow size range around the mean diameter of $120 \mathrm{~nm}$ (Fig. 2a). This agrees well with previously reported data on $\mathrm{CoFe}_{2} \mathrm{O}_{4} / \mathrm{BaTiO}_{3}$ composite nanoparticles partially having the core shell structure [15]. However, separated $\mathrm{CoFe}_{2} \mathrm{O}_{4}$ particles with $40 \mathrm{~nm}$ in diameter could be also found in S1 powder. In spite of the short processing time, SPS resulted in some increasing of the particles size as shown in Fig. 2b for sample $\mathrm{S} 2$. The $\mathrm{BaTiO}_{3}$ grains, seen as bright regions in the SEM image, were distributed in the range $160 \pm 30 \mathrm{~nm}$, while the $\mathrm{CoFe}_{2} \mathrm{O}_{4}$ grains were agglomerated and formed a matrix around the $\mathrm{BaTiO}_{3}$. The grain size $\mathrm{CoFe}_{2} \mathrm{O}_{4}$ 
increased to about $70 \mathrm{~nm}$ (extracted from closeups of SEM images). Annealing of the sample at $900^{\circ} \mathrm{C}$ for 2 hours resulted in the further growth of $\mathrm{BaTiO}_{3}$ grains up to $0.6-0.7 \mu \mathrm{m}$ in diameter as shown for sample S3 in Fig. 2c. Detailed inspection of the $\mathrm{CoFe}_{2} \mathrm{O}_{4}$ matrix revealed that the $\mathrm{CoFe}_{2} \mathrm{O}_{4}$ grains also grew up to about $210 \mathrm{~nm}$. The increasing of grain sizes for both $\mathrm{BaTiO}_{3}$ and $\mathrm{CoFe}_{2} \mathrm{O}_{4}$ is related to the long annealing time and high temperature that facilitate the diffusion process. Nevertheless, the grain sizes remained much smaller in comparison to the sample sintered conventionally (S4), where both $\mathrm{CoFe}_{2} \mathrm{O}_{4}$ and $\mathrm{BaTiO}_{3}$ have grains larger than 1 micron. The morphologies and structures for the different samples are summarized in Table I.

\subsection{ELECTRIC PROPERTIES: A COMPARISON BETWEEN (0-3) AND (3-0) CONNECTIVITY}

Typically, ferroelectric properties of materials are studied by the observation of polarization switching using the Sawyer-Tower method [22]. However, in conductive samples the leakage current can strongly affect the measurements, e.g. resulting in overestimated polarization or coercive field values. Indeed, the total charge in the sample, $Q_{\text {total }}$, measured by the Sawyer-Tower method can be expressed by the following equation:

$$
Q_{\text {total }}(t)=P(E(t)) \cdot A+\int_{0}^{t-c y c l e} I_{\text {leak }}(t) \cdot d t
$$

where $P$ is the polarization, $E$ is the electric field, $A$ is the sample electrode area, $I_{\text {leak }}$ is the leakage current, and $t$ is time. $I_{\text {leak }}(t)$ can be estimated by dividing the applied voltage $V(t)$ by the previously measured resistance of the sample $\left(R_{\text {Leakage }}\right)$, which is assumed to be time independent. Figure 3 shows the electric field dependences of polarization for samples S3 and S4. Both the directly measured hysteresis loops and the curves corrected by subtracting the leakage contribution are shown. One can see that the leakage corrected $P(E)$ dependences retain the hysteretic behavior indicating the ferroelectric character of the $\mathrm{BaTiO}_{3}$ phase as shown in Fig 3a. The leakage 
contribution is large for sample S3 and does not seem to be fully removable assuming a static resistor correction. The leakage is mainly related to the $\mathrm{CoFe}_{2} \mathrm{O}_{4}$ matrix. $\mathrm{CoFe}_{2} \mathrm{O}_{4}$ is a semiconductor and has a conductivity of $10^{-3} \mathrm{~S} / \mathrm{m}$ at room temperature [23], which is 4-5 orders of magnitude larger as for $\mathrm{BaTiO}_{3}$ [24]. In sample S4 with (0-3) connectivity only a very low leakage current was measured due to the isolation of conductive $\mathrm{CoFe}_{2} \mathrm{O}_{4}$ grains by the highly resistive $\mathrm{BaTiO}_{3}$ matrix. Correspondingly, the measured $\mathrm{P}(\mathrm{E})$ curve exactly coincides with the corrected one as shown in Figure 3b. Sample S2 was very leaky so we could not apply a large enough electric field to observe polarization switching. We relate the high conductivity of this sample to some amount of graphite remaining after spark plasma sintering. This residual graphite is removed after annealing.

The dielectric characteristics of different samples are compared in Table 2. Interestingly, the two spark plasma sintered samples show larger permittivity values than the convetionally sintered ceramics, inspite of the smaller grain size of $\mathrm{BaTiO}_{3}$. We attribute this to a significant contribution from the $\mathrm{AC}$ conductivity of the $\mathrm{CoFe}_{2} \mathrm{O}_{4}$ matrix [25]. Indeed the large values of the dielectric permittivity for the SPS prepared samples are accompanied by enhanced dielectric losses (Table 2).

Figure 4 shows PFM images for polished surfaces of ceramic samples. For the sample S2 (Fig. 4b), no PFM active regions were detected, which correlates with the cubic paraelectric structure of $\mathrm{BaTiO}_{3}$ in this case. On the contrary, both samples $\mathrm{S} 3$ and $\mathrm{S} 4$ have regions of a distinct PFM response showing bright and dark contrast in figures $4 \mathrm{~d}$ and $4 \mathrm{f}$. These regions correspond to the ferroelectric $\mathrm{BaTiO}_{3}$ grains. For the SPS sintered and annealed sample (S3) these grains are distributed in the $\mathrm{CoFe}_{2} \mathrm{O}_{4}$ matrix approaching (3-0) connectivity (or potentially (3-3)). The average size of $\mathrm{BaTiO}_{3}$ regions is about $700 \mathrm{~nm}$ which is compatible with the SEM data. The normally sintered sample, S4, shows a completely different morphology: piezoelectrically non-active $\mathrm{CoFe}_{2} \mathrm{O}_{4}$ regions are isolated and distributed in the $\mathrm{BaTiO}_{3}$ matrix. Thus, in this case the 
connectivity (0-3) was formed. To prove the ferroelectric character of the $\mathrm{BaTiO}_{3}$ phase, local PFM hysteresis loops were measured for sample S3. A typical hysteresis loop is shown in Figure 5. The change of the phase of the PFM signal by 180-degree confirms polarization switching at approximately $10 \mathrm{~V}$ (Fig. 5b). The maximal piezoelectric displacement reaches about $0.4 \mathrm{~nm}$ at the maximal applied dc bias $(30 \mathrm{~V})$. Taking into account the amplitude of the probing ac voltage $(8 \mathrm{~V})$, the value of the local longitudinal piezoelectric coefficient can be estimated as $d_{z z} \sim 50 \mathrm{pm} / \mathrm{V}$.

\subsection{MAGNETIC RESPONSE TO A QUASISTATIC EXTERNAL MAGNETIC FIELD}

Figure 6 shows the magnetic field dependences of the mass magnetization measured at room temperature by SQUID magnetometry. The extracted magnetic parameteres are listed in Table 2 . Sample S1, consisting of $\mathrm{CoFe}_{2} \mathrm{O}_{4}-\mathrm{BaTiO}_{3}$ composite nanoparticles with $\mathrm{CoFe}_{2} \mathrm{O}_{4}$ core diameter of about $40 \mathrm{~nm}$, showed a low remanent magnetization $\left(M_{r}\right)$ and a very small coercive field $\left(H_{c}\right)$ in the case of loose, mobile powder. If the powder is fixed, $M_{r}$ and $H_{c}$ increase substantially. Comparison of the different magnetic properties reveal that mobile particles in the powder samples can rotate quite easily yielding an almost vanishing coercive field while the magnetization direction relative to a crystallographic axis does not change. Only if the particles are fixed in the powder sample, which means a particle cannot rotate as a whole any more, the magnetic field dependent magnetization reflects the changes of the magnetization direction with respect to the crystal lattice and can be related to the intrinsic magnetic anisotropies.

For the sample sintered by SPS (S2), the coercive field and remanent magnetization decreased with respect to the fixed powder sample while the saturated magnetization increased. The annealing did not affect the magnetic properties (sample S3). Suprisingly, the SPS prepared samples S2 and S3 showed larger magnetization in comparison to the conventionally sintered ceramic (S4) inspite of much larger $\mathrm{CoFe}_{2} \mathrm{O}_{4}$ grains, about $1-1.5 \mu \mathrm{m}$ in diameter, for the latter. For sample $\mathrm{S} 4$ the saturation 
and remanent magnetization were only $27 \mathrm{Am}^{2} / \mathrm{kg}$ and $10 \mathrm{Am}^{2} / \mathrm{kg}$, respectively. The increased magnetization in SPS sintered ceramics might be due to the higher density of the sample as well as due to the (3-0) connectivity. The conventionally sintered ceramics S4 has (0-3) connectivity with separated $\mathrm{CoFe}_{2} \mathrm{O}_{4}$ grains. In general, the magnetization behavior of $\mathrm{CoFe}_{2} \mathrm{O}_{4}$ is quite complex. The magnetic anisotropy, saturation magnetization and its temperature dependence is related e.g. to the grain size [26,27], cationic disorder [24,28,29,30] and anti-phase boundaries [28,31].

By analysis of the $\mathrm{x}$-ray absorption near edge-structure (XANES) and magnetic circular dichroism (XMCD), changes in the cation distribution can be analysed. Figure 7 shows the spectra of both samples $\mathrm{S} 3$ and $\mathrm{S} 4$ at the $\mathrm{Fe} \mathrm{L}_{3,2}$ and $\mathrm{Co}_{3,2}$ absorption edges. The latter are superimposed by the huge $\mathrm{M}_{5,4}$ absorption lines of Ba. The XANES of Fe shows a double-peak structure at both $\mathrm{L}_{3}$ and $\mathrm{L}_{2}$ absorption edge. At each absorption edge, the relative intensity of the two peaks is related to the valancy of Fe. For sample S3 the spectral shape looks typical for $\mathrm{Fe}^{3+}$. For sample S4 the intensity of the low-energy peak at both edges is enhanced at the expense of the high-energy peak. This indicates a contribution of $\mathrm{Fe}^{2+}$, which may be formed by thermal reduction of $\mathrm{Fe}^{3+}$ ions during the sintering process at $1200^{\circ} \mathrm{C}$ of sample S4 (while sample S3 has been annealed at a lower temperature of $900^{\circ} \mathrm{C}$ ). The XANES of $\mathrm{Co}$ is typical for $\mathrm{Co}^{2+}$ for both samples. The higher absorption intensity of sample S4 is due to a larger Ba absorption peak, which indicates a higher amount of $\mathrm{Ba}$ at the surface compared to sample S3. The XMCD is proportional to the elementspecific magnetization and was measured in a magnetic field of $1.5 \mathrm{~T}$. It is larger for both Fe and Co ions for sample S3 compared to sample S4 in qualitative agreement to the saturation magnetization estimated from SQUID. The W-like shape at the Fe $\mathrm{L}_{3}$ absorption edge is caused by the contributions of Fe ions at different lattice sites. The positive XMCD can be related to Fe ions at tetrahedral lattice sites which are coupled antiferromagnetically to the ions on octahedral lattice sites. Concurrently, the positive XMCD at the $\mathrm{Co} \mathrm{L}_{3}$ absorption edge indicates Co ions at 
tetrahedral lattice sites that should not be there for a perfectly inverted spinel $\mathrm{CoFe}_{2} \mathrm{O}_{4}$. However, it is well known that there exists a cationic disorder in $\mathrm{CoFe}_{2} \mathrm{O}_{4}$ with an inversion factor of $0.68-0.8$ $[29,32]$. As stated by Sawatzky et al [30], the replacement of an $\mathrm{Fe}^{3+}$ ion by a $\mathrm{Co}^{2+}$ ion at tetrahedral lattice sites, will reduce the superexchange interaction significantly leading to a smaller magnetization at room temperature (while at low temperatures it remains largely unchanged). The inversion factor is similar for both samples S3 and S4 and was estimated to be between $0.5-0.7$.

An additional explanation for different saturation magnetization values may be the occurrence of anti-phase boundaries between cation lattices that are displaced in a way that the system remains monocrystalline. These displacements cannot be seen in XANES or the spectral shape of XMCD, but can strongly reduce the saturation magnetization since the spin couplings are reversed across the boundary leading to oppositely magnetized regions [31].

An element-specific magnetic hysteresis curve at the $\mathrm{Fe}_{3}$ absorption edge was measured with the surface-sensitive the total electron yield (TEY) detection mode. The magnetic field direction was the same as for the magnetoelectric coupling measurements by $\mathrm{x}$-ray linear dichroism (XLD) that will be presented below (30 off the sample surface normal), i.e. with its main component perpendicular to the sample surface for a reliable correlation to the XLD data. The results are shown in Fig. 8 for samples S3 and S4. Note that measurements at the Co $\mathrm{L}_{3}$ absorption edge are disturbed by the huge $\mathrm{Ba}_{5}$ absorption line close in energy (Fig. 7). We expect that due to the exchange coupling, element-specific magnetic hystereses of $\mathrm{Fe}$ and $\mathrm{Co}$ are similar in $\mathrm{CoFe}_{2} \mathrm{O}_{4}$.

There are three obvious differences of these magnetisation curves compared to the SQUID data: (i) larger coercive fields, (ii) larger slope at high magnetic fields, and (iii) larger difference in saturation magnetizations of sample S3 and S4. All the points (i)-(iii) are attributed to the surfacesensitivity of the XAS. Note that point (ii) can be related to the surface-sensitivity of XAS only, 
since the high-field slope caused by spin canting, cationic disorder, and anti-phase boundaries is expected to be isotropic in any case.

An additional hint that the differences are related to surface effects is the fact that the large coercive field is similar to the one of the fixed powder sample that has a large surface fraction either. Beside the differences of the (spin) structure at the surface compared to the bulk fraction of the sintered ceramics, a larger magnetic anisotropy caused by magnetostriction can also occur at the surface since the magnetostriction of $\mathrm{CoFe}_{2} \mathrm{O}_{4}$ is less impeded by the surrounding $\mathrm{BaTiO}_{3}$.

\subsection{MAGNETOELECTRIC COUPLING}

Figure 9a shows the the ME induced magnetization as function of the superimposed dc magnetic field for the annealed SPS ceramics S3 and the normally sintered ceramics S4. Both curves have the shape, which is often observed for composite multiferroics, with a pronounced peak of the ME response at a certain field. This peak corresponds to the maximal piezomagnetic coefficient, $q$, which in turn correlates to the maximal change of magnetostriction with respect to the magnetic field, $q=\mathrm{d} \lambda / \mathrm{d} H$ [5]. The maximum value of the ME induced magnetization was recorded at $0.1 \mathrm{~T}$ and about $0.2 \mathrm{~T}$ for samples $\mathrm{S} 3$ and $\mathrm{S} 4$, respectively. The observed shift of the maximal ME response can be attributed to the different $\mathrm{CoFe}_{2} \mathrm{O}_{4}$ grain sizesin samples $\mathrm{S} 3$ and $\mathrm{S} 4$ which causes different field dependencesof magnetostriction [33]. The sign of the magnetoelectric response changes for sample S3 above $0.3 \mathrm{~T}$, while it remains positive for sample $\mathrm{S} 4$ in the field range studied.

Figure $9 \mathrm{~b}$ shows the ME induced magnetization as a function of the applied ac voltage. The dependences are (nearly) linear for both samples. From the slope of the curves the value of the converse magnetoelectric coefficient, $\alpha_{\mathrm{C}}$, can be estimated according the relation, $\mu_{o} H_{a c}=\alpha_{C} E_{a c}$, with $E_{a c}$ being the amplitude of the applied ac electric field. The best fit gives $\alpha_{C} \sim 1 \mathrm{ps} / \mathrm{m}$ and 25 
ps/m for samples S3 and S4, respectively. Such a big difference might be due to several reasons. First of all the higher resistivity of the (0-3) connected sample S4 as compared to the (3-0) connected sample $\mathrm{S} 3$ ensures that a larger electric field can be applied to the $\mathrm{BaTiO}_{3}$ grains during poling and measurement.This will result in more effective poling of the ferroelectric component and hence in enhancement of the piezoelectric effect. The larger electric field during ME measurements will again result in a larger piezoelectric strain at the $\mathrm{BaTiO}_{3} / \mathrm{CoFe}_{2} \mathrm{O}_{4}$ interface and hence in a stronger ME response. Due to smaller $\mathrm{BaTiO}_{3}$ grains in sample $\mathrm{S} 3$ one can expect a reduction of the piezoelectric coefficient due to the size effect [34] and correspondingly a weaker ME coupling. Similarly, the smaller size of $\mathrm{CoFe}_{2} \mathrm{O}_{4}$ grains in sample $\mathrm{S} 3$ can be a reason of the smaller piezomagnetic coefficient [33], which again will result in a weaker ME coupling.

X-ray absorption spectroscopy and its associated x-ray linear dichroism (XLD) at the $\mathrm{Ti}_{3,2}$ absorption edges have been shown to be a valuable tool to investigate the magnetic field-dependent electric polarisation [20]. Measurement of TEY is a possibility to detect the coupling in a surface sensitive mode since the information depth is limited by the electron escape depth, i.e. a few nanometres. In Fig. 10, the x-ray absorption near-edge structure (XANES) and XLD at the Ti L 3,2 absorption edges of samples S3 and S4 are shown for two different field values. The spectral shape of the XANES can be described by four main peaks (two at the L3 absorption edge and two at the L2 absorption egde) that reflect the crystal field splitting of the final $3 \mathrm{~d}$ states. The two small leading peaks at photon energies between $456 \mathrm{eV}$ and $458 \mathrm{eV}$ are typical for a $2 \mathrm{p}^{6} 3 \mathrm{~d}^{0} 2 \mathrm{p} 5^{3} \mathrm{~d}^{1}$ transition as has been shown by multiplet calculations. In agreement to other experimentally obtained spectra of $\mathrm{Ti}$ ions in $\mathrm{BaTiO}_{3}$ in the ferroelectric tetragonal phase, the pre-peaks are sharp and clearly visible for sample S3. For sample S4 they are smeared out and slightly shifted to energies closer to the first main peak. This may be explained by reduced intra-atomic Coulomb and exchange parameters for the Ti ions [35], which is also indicated by a change of the $\mathrm{L}_{2} / \mathrm{L}_{3}$ branching 
ratio which is slightly enhanced for sample S4 compared to S3. A reduction of the intra-atomic interactions may be due to covalent screening effects. In addition, the energy positions of the main peaks indicate a reduced crystal field splitting. In summary, the XANES of samples S3 and S4 indicate the typical bulk-like $\mathrm{BaTiO}_{3}$ structure at room teperature of sample S3 while sample S4 shows significant deviations probably correlated to a more covalent character of the bondings of $\mathrm{Ti}$ ions to their neighboring ions.

The XLD of both samples S3 and S4 is shown in Fig. 7 for two different values of the external magnetic field, i.e. $0.15 \mathrm{~T}$ and $1.0 \mathrm{~T}$, respectively. The magnetic field was applied with its main component perpendicular to the sample surface. The electric field vector of the incoming $\mathrm{x}$ rays was either parallel to the magnetic field (horizontal polarisation) or perpendicular (vertical polarisation). For both samples, it can be seen that the XLD amplitude depends on the magnetic field value. However, while for sample S3 the XLD is larger for the small magnetic field, sample S4 exhibits the opposite behaviour. To study this in more detail, XLD measurements have been analysed for different magnetic fields between $-0.02 \mathrm{~T}$ and $1.5 \mathrm{~T}$. In Fig. 11, the magnetic field dependence of the square root of XLD amplitude is shown, that is a measure of the electric polarisation acoording to [36]:

$I=\left\langle P^{2}\right\rangle\left(1-\cos ^{2} \theta_{P, E}\right)$

where $\theta_{P, E}$ is the angle between electric polarisation of the sample and the electric field vector of $\mathrm{x}^{-}$ rays. Assuming that the electric polarisation is either parallel or perpendicular to the magnetic field applied, the difference between the absorption signals for horizontally and vertically polarised $\mathrm{x}$ rays is proportional to the statistical average of the squared local polarisation. The data have been normalized to the value obtained in a small magnetic field of $0.02 \mathrm{~T}$. However, one should note that in small magnetic fields applied $\left(-0.02 \mathrm{~T} \leq \mu_{0} \mathrm{H}_{\mathrm{ext}} \leq 0.02 \mathrm{~T}\right)$, the measurement of the TEY is error 
prone. The XLD asymmetries used for the normalisation are $2.8 \%$ for sample $\mathrm{S} 3$, which is close to the asymmetry reported for a epitaxial $\mathrm{CoFe}_{2} \mathrm{O}_{4}-\mathrm{BaTiO}_{3}$ nanocomposite with an oriented c-axis perpendicular to the sample surface (2.2\%) [20] and 1.1\% for sample S4. The different values for the XLD asymmetry correspond to a larger electric polarisation of sample S3 with respect to sample S4. For sample $\mathrm{S} 3$, the maximum polarisation is obtained at $\mu_{0} \mathrm{H}_{\mathrm{ext}}=0.15 \mathrm{~T}$ and is decreasing with further increasing value of the magnetic field until it reaches a minimum at about 1.0T. For higher magnetic fields, it is again slightly increasing following the shape of a typical magnetostriction curve of $\mathrm{CoFe}_{2} \mathrm{O}_{4}$ [37-39]. Interestigly, for large magnetic fields, the polarisation is reduced to only about $90 \%$ of the value at very low magnetic fields. This may be explained in a simplistic picture by the compression of the $\mathrm{BaTiO}_{3}$ grains in the $\mathrm{CoFe}_{2} \mathrm{O}_{4}$ matrix along the field direction due to the negative magnetostriction of $\mathrm{CoFe}_{2} \mathrm{O}_{4}$ along the magnetic field direction whose absolute value is twice as large as the positive magnetostriction in the sample plane yielding a smaller tetragonal distortion of the $\mathrm{BaTiO}_{3}$ unit cell.

For sample $\mathrm{S} 4$ the maximum polarisation is obtained between $0.5 \mathrm{~T}$ and $1.0 \mathrm{~T}$ and is again slightly decreasing for further increasing external magnetic fields. In contrast to sample S3, the polarisation at high magnetic fields is always larger than the one at the lowest field of $0.02 \mathrm{~T}$. This can be explained in the same simplistic picture by the different connectivity: While in sample S3 the $\mathrm{CoFe}_{2} \mathrm{O}_{4}$ matrix compresses the $\mathrm{BaTiO}_{3}$ grains in a way that the unit cell becomes more cubic, in sample S4 the grains shrink along the field direction and expand in the perpendicular directions forcing a larger tetragonal distortion of the $\mathrm{BaTiO}_{3}$ unit cell with its long axis parallel to the applied magnetic field.

To compare the magnetic field dependence of the electric polarization obtained from XLD with measurements the of electrically induced magnetization using ac-SQUID susceptometry, one has to take into account that in the first case the integral magnetoelectric effect is measured, while in the 
second case the differential ME response is probed. The XLD signal tracks the magnetic field induced strain, i.e. the field dependence of the magnetostriction. While, the electrically induced magnetization is $M_{M E} \sim q_{l} d_{l} E$ where $E$ is weak probing electric field, $d_{l}$ is the piezoelectric coefficient of the ferroelectric phase, and $q_{l}$ is a piezomagnetic coefficient of the magnetic phase [40]. The latter can be defined as $q_{l}=\delta \lambda / \delta H$, where $\lambda$ is the magnetostriction [41]. Thus, the magnetic field depence of ME effect measured by SQUID (Fig. 9) should behave as a derivative of magnetic field dependence measured by XLD (Fig. 11). Indeed, for sample S3 we observe that Ti polarization reaches the maximum at approximately $0.3 \mathrm{~T}$ and than decreases, which behaviour matches well the change of the sign of the ME response by the SQUID measurements. For sample S4 the XLD signal is saturated around $1 \mathrm{~T}$, correspondigly the ME induced magnetization drops to zero.

\section{Conclusion}

In conclusion, a (3-0) composite of cobalt ferrite and barium titanate was successfully synthesized using the spark plasma sintering method and compared to a conventionally sintered (0-3) reference sample. An improvement of the magnetic properties in comparison to the conventionally sintered ceramics was achieved, while the good ferroelectric characteristics were retained. The converse magnetoelectric effect is weaker in the SPS sample than in the conventionally sintered one. This is mainly related to the larger leakage current in the former sample preventing efficient electric poling. On the microscopic scale it was found by x-ray absorption spectroscopy that the spinel inversion factor of $\mathrm{CoFe}_{2} \mathrm{O}_{4}$ is similar for both preparation techniques. The changes in the magnetic properties can be related to different grain sizes, anti-phase boundaries, and interface hybridization effects. In addition, a good qualitative agreement between the magnetic field dependence of the electric 
polarization obtained from XLD with measurements of the electrically induced magnetization using ac-SQUID susceptometry was obtained.

\section{ACKNOWLEDGMENT}

M. Etier thanks the DAAD-GRISEC program (Grant 50750877) for the financial support. H. Trivedi thanks the European Commission for support within FP7 Marie Curie Initial Training Network "Nanomotion" (grant agreement $n^{\circ}$ 290158). Support through the BMBF (05 ES3XBA/5) and Deutsche Forschungsgemeinschaft via Forschergruppe 1509 "Ferroic Functional Materials" are acknowledged. Schwerpunktprogramm 1681 "Feldgesteuerte Partikel-Matrix Wechselwirkungen" and Stiftung Mercator (MERCUR) is acknowledged.

S. Salamon would like to thank U. von Hörsten and P. Borisov for their expert technical assistance and improvements made to the SQUID setup. E. Weschke and E. Schierle's support during synchrotron beamtime is gratefully acknowledged.

\section{REFERENCES}

1. Y. Wang, D. Grey, D. Berry, J. Gao, M. Li, J. Li, and D. Viehland, Adv. Mater. 23 (2011) 4111-4114.

2. S. Marauska, R. Jahns, C. Kirschof, M. Klaus, E. Quandt, R. Knöchel, and B. Wagner, Sensor. Actuat. A-Phys. 189 (2013) 321-327.

3. S. Dong, J. Zhai, J. F. Li, D. Viehland, and S. Priya, Appl. Phys. Lett. 93 (2008) 103511-103513.

4. S. Agarwal, O.F. Caltun, and K. Sreenivas, Sol. St. Com. 152 (2012) 1951-1955.

5. V. V. Shvartsman, F. Alawneh, P. Borisov, D. Kozodaev, and D. C. Lupascu, Smart. Mater. Struct. 20 (2011) 075006-075012.

6. J.-P. Zhou, L. Lv, Q. Liu, Y.-X. Zhang, and P. Liu, Sci. Technol. Adv. Mater. 13 (2012) 045001-045013.

7. M. H. Frey and D. A. Payne, Phys. Rev. B. 54[5] (1996) 3158-3168.

8. K. Maaz, A. Mumtaz, S. K. Hasanain, and A. Ceylan. Magn. Magn. Mater. 308 (2007) 289-295.

9. M. T. Buscaglia, V. Buscaglia, M. Viviani, J. Petzelt, M. Savinov, L. Mitoseriu, A. Testino, P. Nanni, C. Harnagea, Z. Zhao, and M. Nygren, Nanotechnology. 15 (2004) 1113-1117. 
10. X. Deng, X. Wang, H. Wen, L. Chen, L. Chen, and L. Li, Appl. Phys. Lett. 88 (2006) 252905-252908.

11. D. Ghosh, H. Han, J. C. Nino, G. Subhash, and J. L. Jones, J. Am. Ceram. Soc. 95 (2012) 2504-2509.

12. R. E. Newnham, D. P. Skinner, and L. E. Cross, Mat. Res. Bull. 13 (1978) 525-536.

13. G. A. Smolenskii and I. E. Chupis, Sov. Phys. Usp. 25 (1982) 475-493.

14. V. M. Petrov and V. V. Gagulin, Inorg. Mater. 37 (2001) 93-98.

15. M. Etier, Y. Gao, V. V. Shvartsman, A. Elsukova, J. Landers, H. Wende, and D. C. Lupascu, Ferroelectrics, 438 (2012) 115-122.

16. Y. Gao, V. V. Shvartsman, A. Elsukova, and D. C. Lupascu, J. Mater. Chem. 22 (2012) 17573-17583.

17. Y. Gao, V. V. Shvartsman, D. Gautam, M. Winterer, and D. C. Lupascu, J. Am. Ceram. Soc. 97 (2014) 21392146.

18. G. V. Duong, R. Groessinger, M. Schoenhart, and D. Bueno-Basques. J. Magn. Magn. Mater. 316 (2007) 390393.

19. P. Borisov, A. Hochstrat, V. V. Shvartsman, and W. Kleemann, Rev. Sci. Instrum. 78 (2007) 106105-106108.

20. C. Schmitz-Antoniak, D. Schmitz, P. Borisov, F. M. F. de Groot, S. Stienen, A. Warland, B. Krumme, R. Feyerherm, E. Dudzik, W. Kleemann, and H. Wende, Nature Commun. 4 (2012) 3051-3059.

21. H.-I. Hsiang and F.-S. Yen, J. Am. Ceram. Soc. 79 (1996) 1053-1060.

22. C. B. Sawyer and C. H. Tower, Phys. Rev. 35 (1930) 269-273.

23. C. O. Augustin, L. K. Srinivasan, P. Kamaraj, and A. Mani, J. Mater. Sci. Technol. 12 (1996) 417-420.

24. A. Yu. Kudzin, Izvestija VUZ. Fizika (Sovjiet Physics Journal), 10 (1967) 124-126.

25. I. H. Gul and A. Maqsood, J. All. Compoud. 465 (2008) 227-231.

26. M. Etier, V. V. Shvartsman, F. Stromberg, J. Landers, H. Wende, and D. C Lupascu, Mater. Res. Soc. Symp. Proc. Vol. 1398 (2012), DOI: 10.1557/opl.2012.699.

27. K. V. P. M. Shafi, A. Gedanken, R. Prozorov, and J. Balogh, Chem. Mater. 10 (1998) 3445-3450.

28. B. Y. Wang, S. B. Singh, Y. C. Shao, Y. F. Wang, C. H. Chuang, P. H. Yeh, J. W. Chiou, C. W. Pao, H. M. Tsai, H. J. Lin, J. F. Lee, C. Y. Tsai, W. F. Hsieh, M.-H. Tsai and W. F. Pong, RSC Adv. 3 (2013) 7884-7893.

29. D. Carta, M.F. Casula, A. Falqui, D. Loche, G. Mountjoy, C. Sangregorio, and A. Corrias, J. Phys. Chem. C 113 (2009) 8606-8615.

30. G. A. Sawatzky, F. van der Woude, and A. H. Morrish, J. Appl. Phys. 39 (1968) 1204-1206. 
31. D. T. Margulies, F. T. Parker, M. L. Rudee, F. E. Spada, J. N. Chapman, P. R. Aitchison, and A. E. Berkowitz, Phys. Rev. Lett. 79 (1997) 5162-5165.

32. A. S. Vaingankar, B. V. Khasbardar, and R. N. Patil, J. Phys. F: Met. Phys. 5[34] (1981) 387-389.

33. S.D. Bhame and P.A. Hoy, Sensors and Actuators A, 137 (2007) 256-261.

34. Y. Huan, X. Wang, J. Fang, and L. Li, J. Am. Ceram. Soc. 96 (2013) 3369-3371.

35. F. M. F. de Groot, J. C. Fuggle, B. T. Thole, and G. A. Sawatzky, Phys. Rev. B. 41 (1990) 928-937.

36. S. Polisetty, J. Zhou, J. Karthik, A. R. Damodaran, D. Chen, A. Scholl, L.W. Martin, and M. Holcomb, J. Phys.: Cond. Matter 24 (2012) 245902-245909.

37. R. W. McCallum, K. W. Dennis, D. C. Jiles, J. E. Snyder, and Y. H. Chen, Low Temp. Phys. 27 (2001) $266-$ 274

38. S. D. Bhame and P. A. Joy, J. Appl. Phys. 100 (2006) 113911-113915.

39. Y. X. Zheng, Q. Q. Cao, C. L. Zhang, H. C. Xuan, L. Y. Wang, D. H. Wang, and Y. W. Du, J. Appl. Phys. 110 (2011) 043908-043915.

40. T. Wu, C.-M. Chang, T.-K. Chung, and G. Carman, IEEE. Trans. Magn. 45 (2009) 4333-4336.

41. G. Srinivasan, E. T. Rasmussen, and R. Hayes, Phys. Rev. B. 67 (2003) 014418-014428. 


\section{LIST OF FIGURES}

FIGURE 1. XRD spectra of $\mathrm{CoFe}_{2} \mathrm{O}_{4}-\mathrm{BaTiO}_{3}$ (50-50) composites for the samples (a) powder calcinated at $750^{\circ} \mathrm{C}$ for 15 minutes (sample S1), (b) ceramic sintered by SPS at $1000^{\circ} \mathrm{C}$ for 5 minutes (sample S2), and (c) ceramic SPS sample annealed at $900^{\circ} \mathrm{C}$ for 2 hours (sample S3). Diffraction peaks corresponding to the $\mathrm{BaTiO}_{3}$ and $\mathrm{CoFe}_{2} \mathrm{O}_{4}$ phases are marked by squares and circles, correspondingly. The panel on the right (d) shows an enlarged view of the diffraction peaks corresponding to the crystallographic (112) and (211) planes of $\mathrm{BaTiO}_{3}$ for each sample.

FIGURE 2. SEM images obtained using the backscattered electron mode of samples (a) S1 (b) S2 and (c) S3. The insets show the $\mathrm{BaTiO}_{3}$ particle size distributions for each sample.

FIGURE 3. Room temperature polarization-electric field hysteresis loops measured at $100 \mathrm{~Hz}$ as measured (black symbols) and after subtracting the (static) leakage contribution (red symbols) for samples (a) S3 and (b) S4.

FIGURE 4. PFM images of the $\mathrm{CoFe}_{2} \mathrm{O}_{4}-\mathrm{BaTiO}_{3}$ composites showing topography and vertical PFM response of samples S2, S3 and S4 as indicated.

FIGURE 5. Local piezoresponse hysteresis loop: (a) amplitude and (b) phase, in a $\mathrm{BaTiO}_{3}$ grain for sample $\mathrm{S} 3$.

FIGURE 6. Room temperature magnetization hysteresis loops for the different $\mathrm{BaTiO}_{3}-\mathrm{CoFe}_{2} \mathrm{O}_{4}$ samples from the SQUID. (a) Shows fixed powder versus mobile powder sample S1, (b) all sintered samples S2, S3, S4.

FIGURE 7. X-ray absorption near edge structure and $\mathrm{x}$-ray magnetic circular dichroism in a magnetic field of $1.5 \mathrm{~T}$ at a temperature of $290 \mathrm{~K}$ for sample S3 (black) and S4 (red line).

FIGURE 8: Magnetic field dependence of the XMCD asymmetry at the $\mathrm{Fe} \mathrm{L}_{3}$ absorption edge as a measure of the magnetization at a temperature of 290K for sample S3 (black) and S4 (red line).

FIGURE 9. (a) DC magnetic field dependence of the ME induced magnetization at $T=285 \mathrm{~K}, V_{a c}=200 \mathrm{~V}$, and $f_{a c}=3$ $\mathrm{Hz}$ for samples S3 (open circles) and S4 (solid circles) (b) AC electric field dependence of the ME induced magnetization at $T=285 \mathrm{~K}, H_{d c}=0.15 \mathrm{~T}$, and $f_{a c}=3 \mathrm{~Hz}$ for the samples S3 (open circles) and $\mathrm{S} 4$ (solid circles).

FIGURE 10: XANES and XLD at the $\mathrm{Ti}_{3,2}$ absorption edges of sample $\mathrm{S} 3$ for two different values of the external magnetic field applied as indicated. Measurements were performed at a temperature of $290 \mathrm{~K}$.

FIGURE 11: Magnetic field dependence of electric polarization as extracted from XLD measurements at the Ti $\mathrm{L}_{3,2}$ absorption edges for sample S3 (black) and S4 (red symbols) at temperature of 290K. 


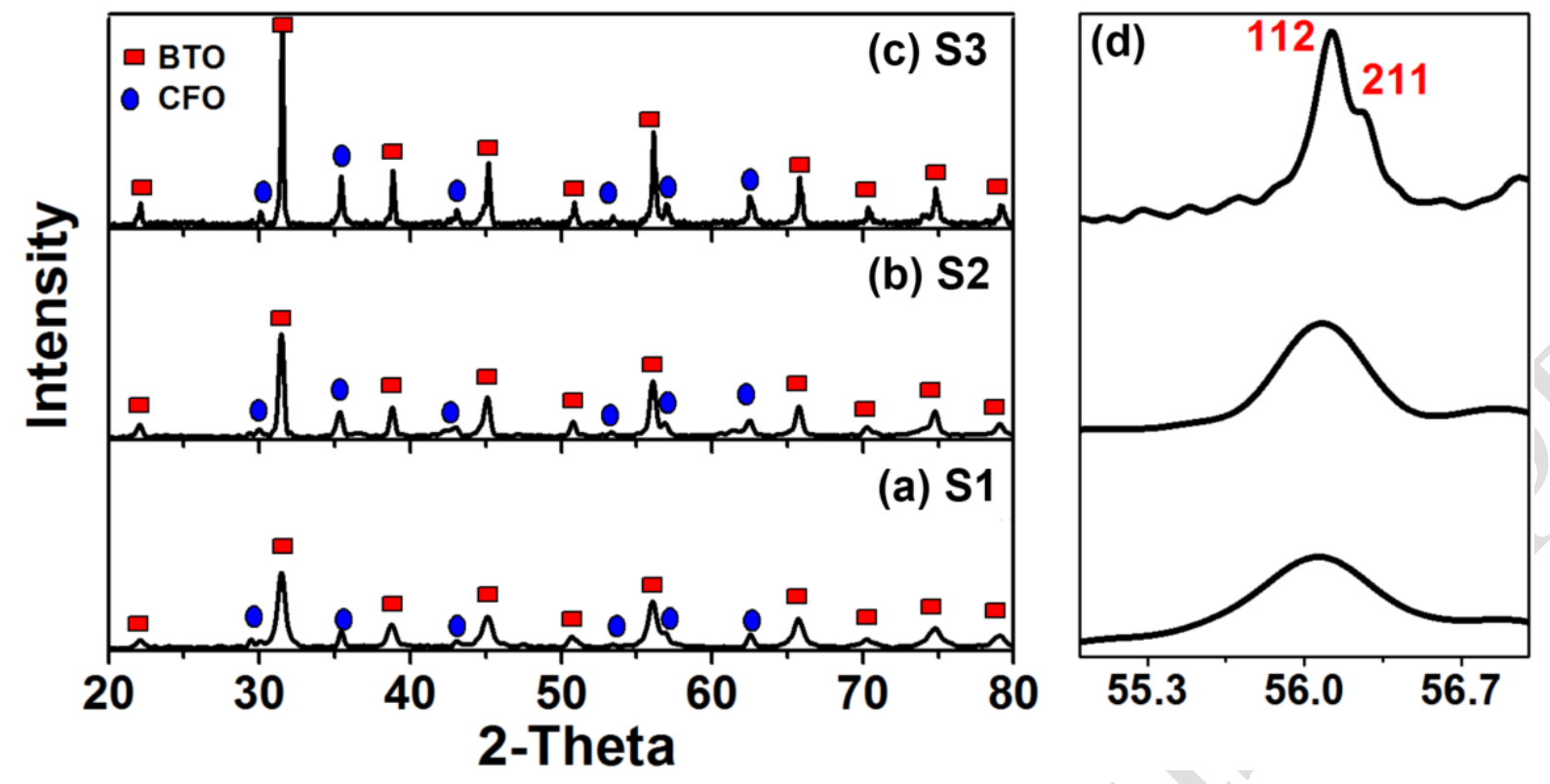

Figure (1) 


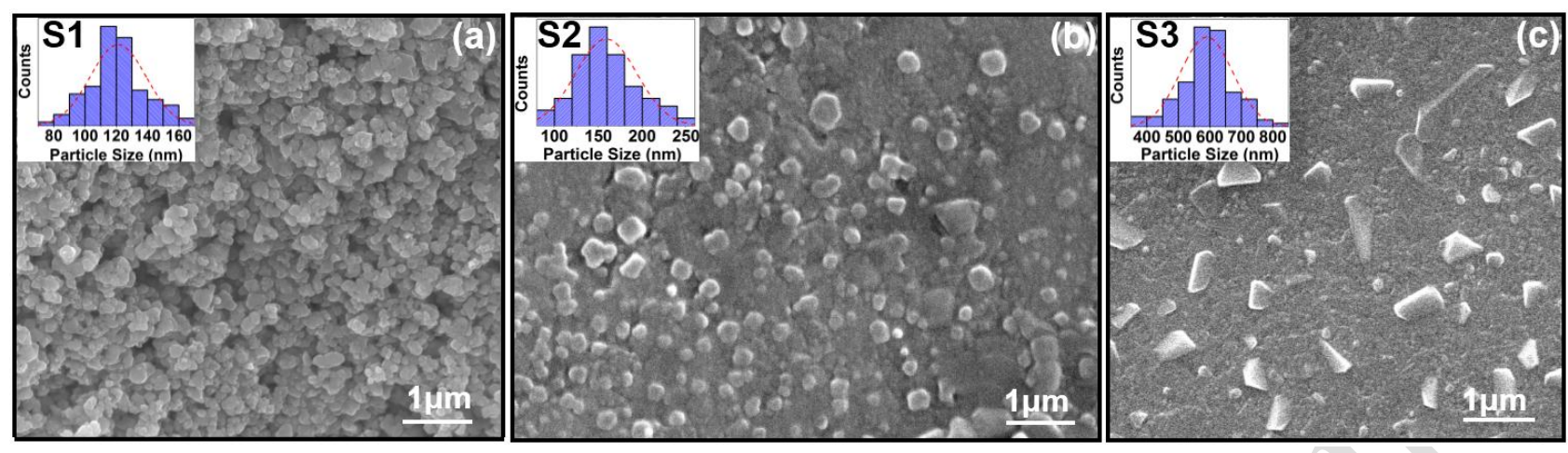

Figure (2) 

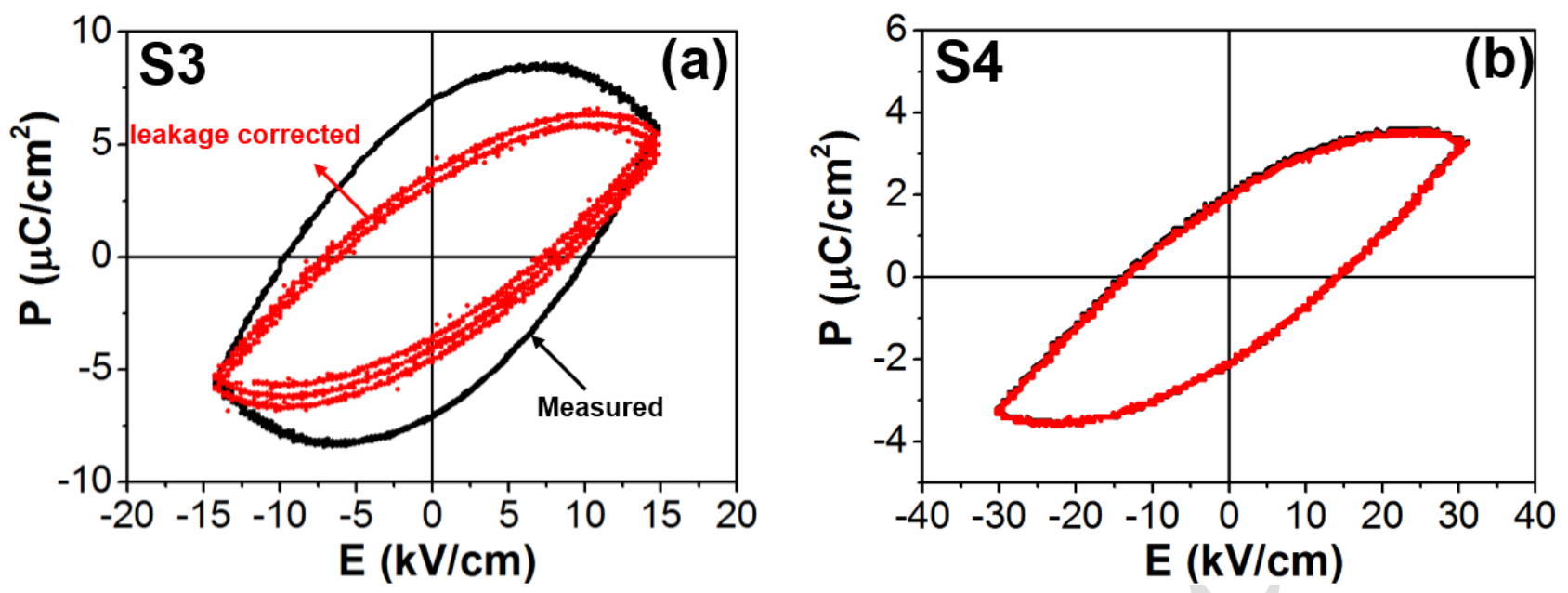

Figure (3) 


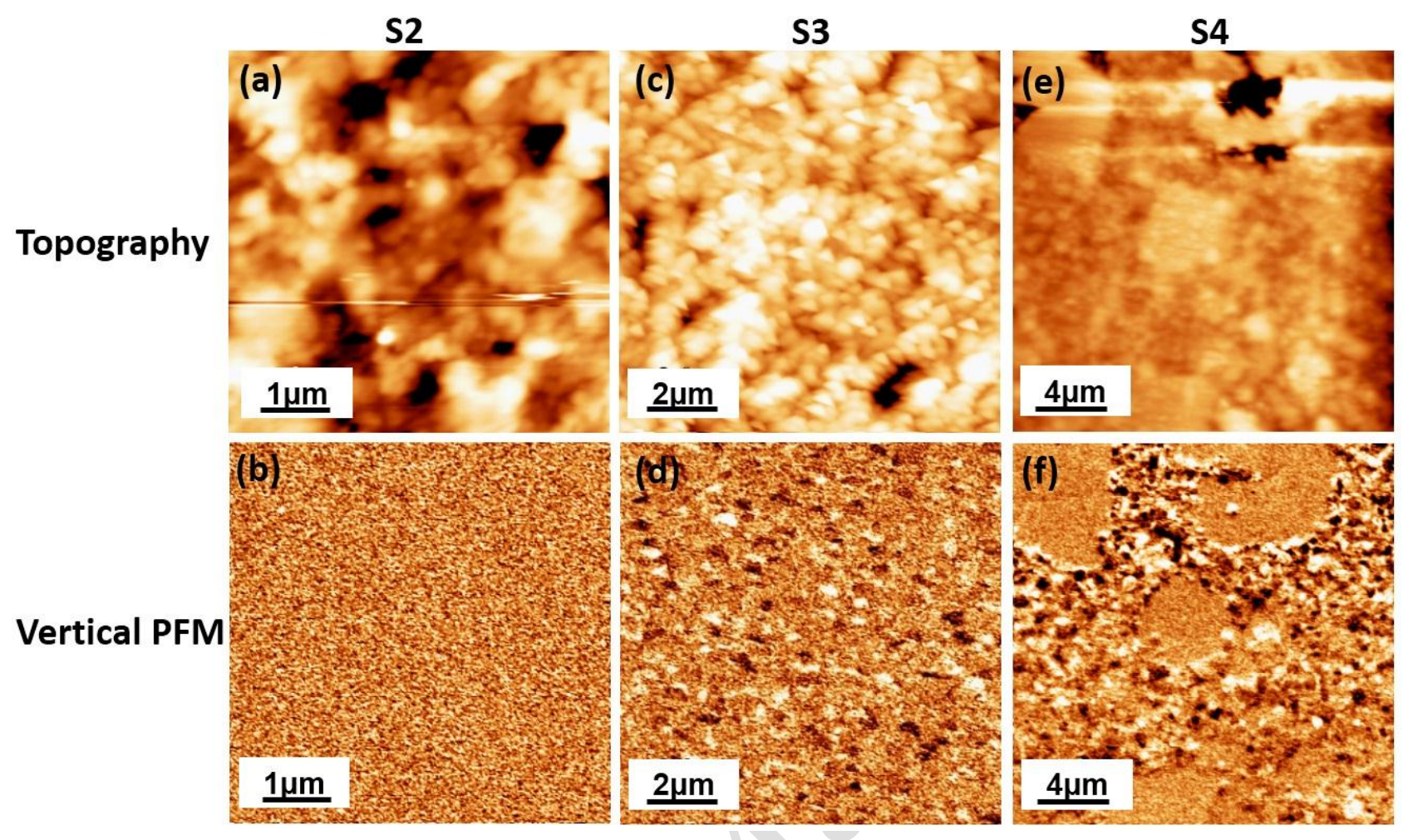

Figure (4) 

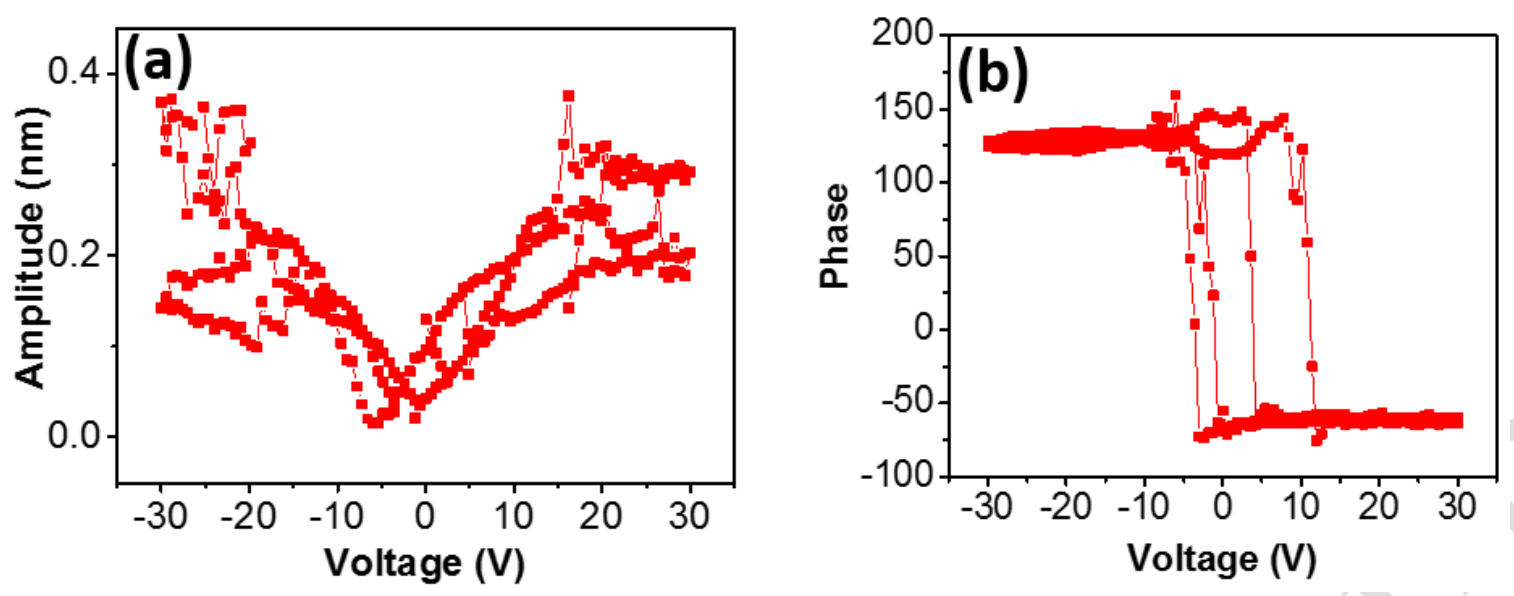

Figure (5) 

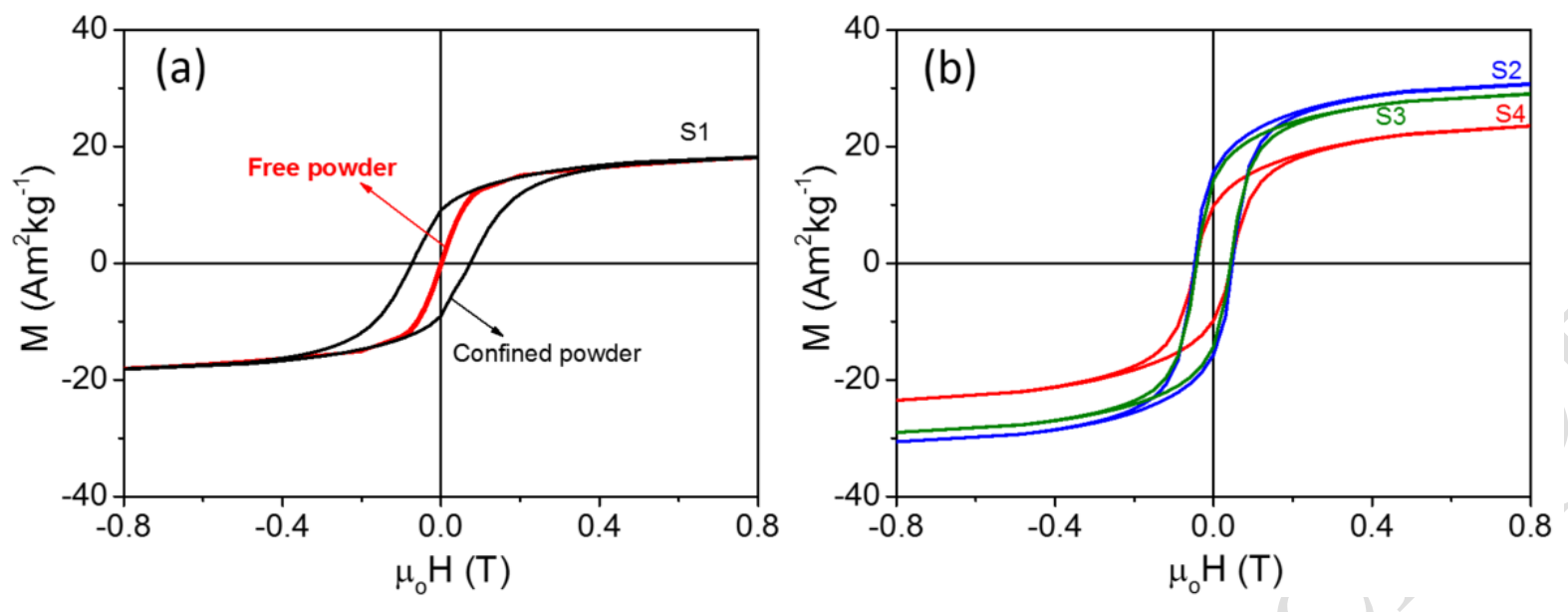

Figure (6) 


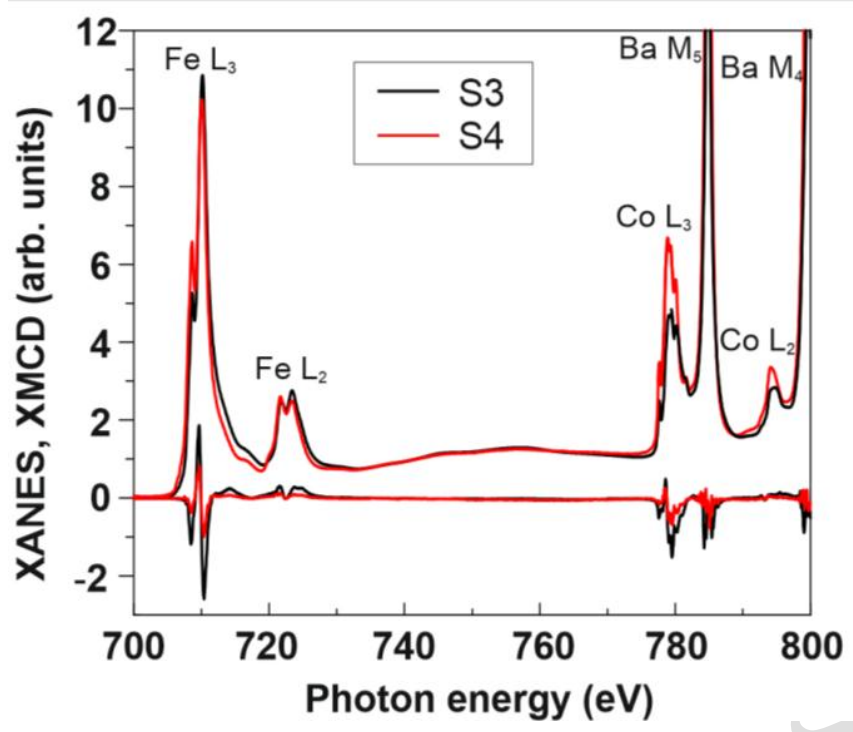

Figure (7) 


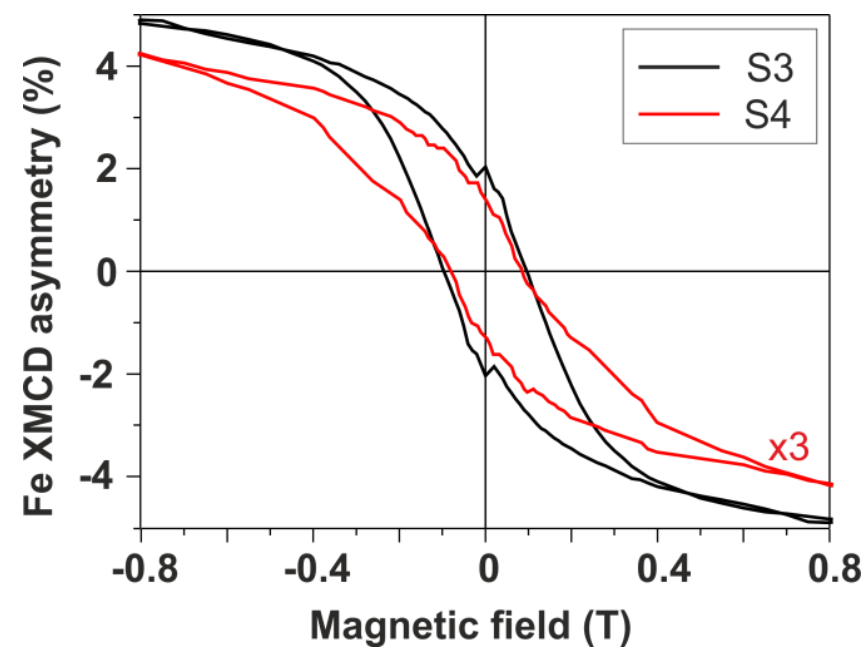

Figure (8) 


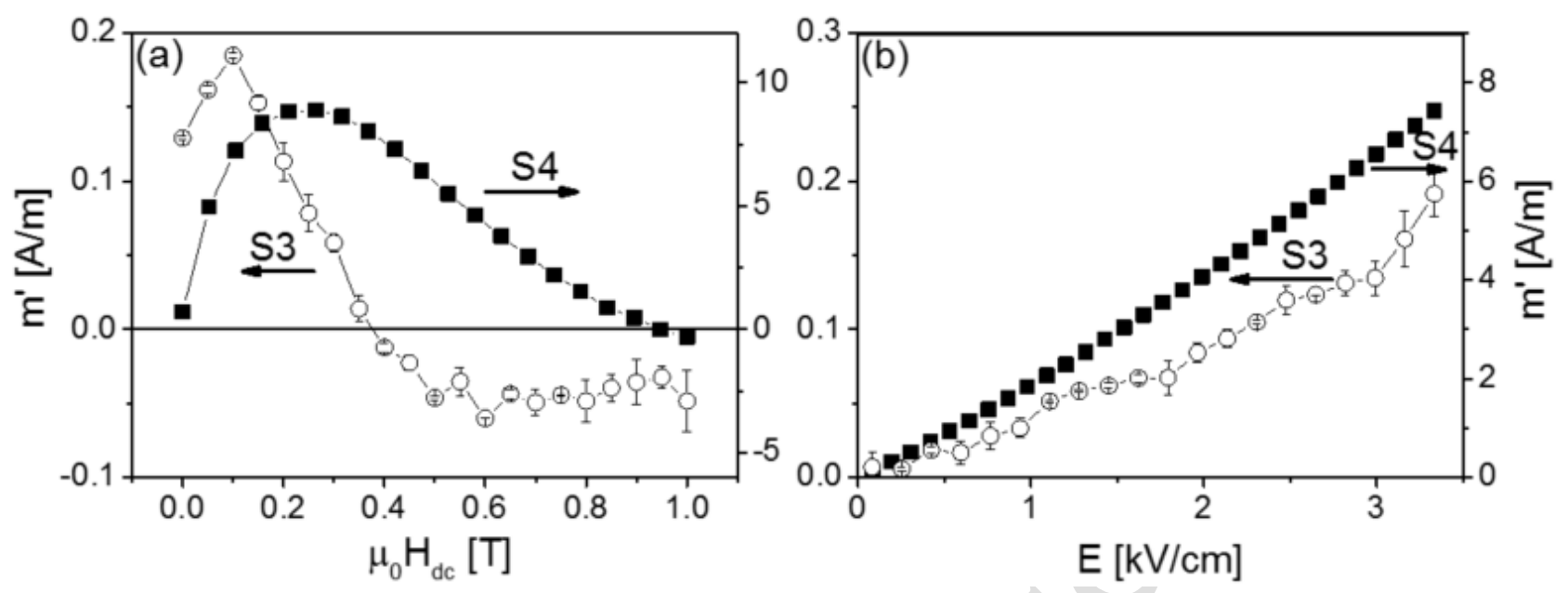

Figure (9) 


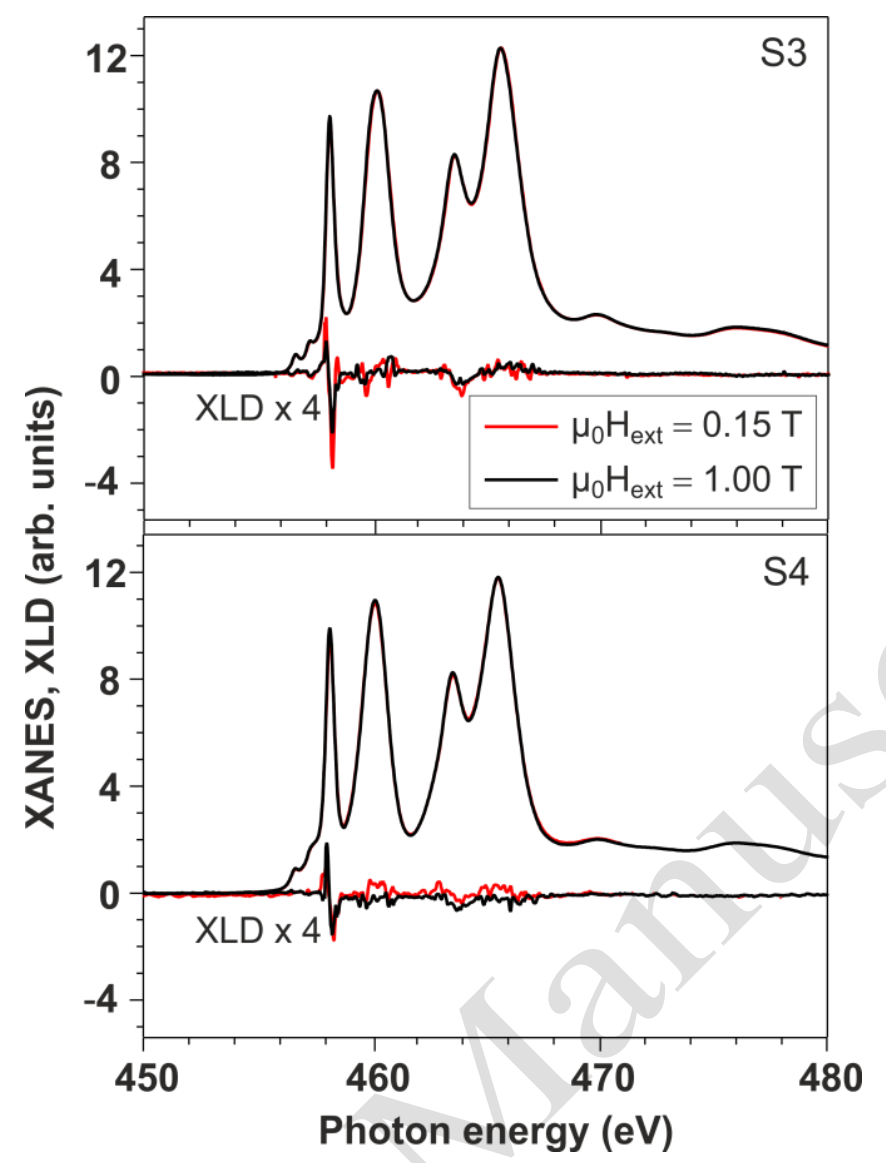

Figure (10) 


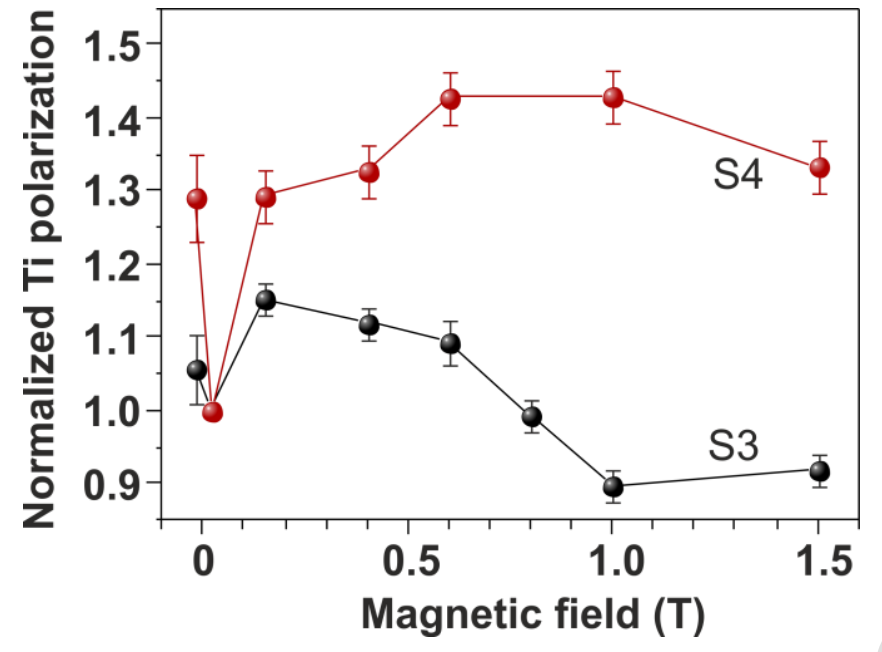

Figure (11) 


\section{LisT OF TABLES}

TABLE 1. Short description of samples S1-S4, morphologies and structural properties.

\begin{tabular}{|r|c|c|c|c|}
\hline & S1 & S2 & S3 & S4 \\
\hline Description & Powder & SP sintered & $\begin{array}{c}\text { SP sintered }+ \\
\text { annealed }\end{array}$ & $\begin{array}{c}\text { conventionally } \\
\text { sintered }\end{array}$ \\
\hline connectivity & - & $3-0$ & $3-0$ & $0-3$ \\
\hline $\mathrm{BaTiO}_{3}$ structure & cubic & cubic & tetragonal & tetragonal \\
\hline $\mathrm{BaTiO}_{3}$ grain size $(\mathrm{nm})$ & $40($ shell) -120 & $160 \pm 30$ & $650 \pm 50$ & $1000-2500$ \\
\hline $\mathrm{CoFe}_{2} \mathrm{O}_{4}$ grain size $(\mathrm{nm})$ & 40 (core) & $70 \pm 10$ & $210 \pm 25$ & $1000-1500$ \\
\hline
\end{tabular}

TABLE 2. Room temperature dielectric and magnetic properties for the different samples

\begin{tabular}{|c|c|c|c|c|c|c|}
\hline Sample & $\begin{array}{c}\varepsilon^{\prime} \\
\text { at } 100 \mathrm{kHz}\end{array}$ & $\begin{array}{c}\tan \delta \\
\text { at } 100 \mathrm{kHz}\end{array}$ & $\begin{array}{c}M_{s} \\
\left(\mathrm{Am}^{2} \mathrm{~kg}^{-1}\right)\end{array}$ & $\begin{array}{c}M_{r} \\
\left(\mathrm{Am}^{2} \mathrm{~kg}^{-1}\right)\end{array}$ & $M_{r} / M_{s}$ & $\begin{array}{c}\mu_{0} H_{c} \\
(\mathrm{mT})\end{array}$ \\
\hline S1 (confined) & - & - & 20 & 9 & 0.45 & 75 \\
\hline S2 & 175 & 0.25 & 32.5 & 15.5 & 0.48 & 47 \\
\hline S3 & 208 & 0.47 & 32 & 15 & 0.47 & 45 \\
\hline S4 & 73 & 0.033 & 27 & 10 & 0.37 & 45 \\
\hline
\end{tabular}

